\section{Concluding remarks}

Although a role for complement in tissue damage in diverse clinical situations is now widely accepted ${ }^{9}$, no suitable therapeutic strategies for inhibiting complement have, until recently, been available. Basic research on membrane regulatory molecules has focused attention on a new panel of agents that might prove effective in therapy. With the enormous effort now being invested in the identification of active sites in these regulators, and in the design of moreactive forms for use in vivo, it is likely that we will see inhibition of complement becoming an accepted clinical procedure. Perhaps the first instances where these inhibitors will find a niche is in the prevention of the iatrogenic injury that often accompanies cardiopulmonary bypass, as well as in other situ- ations where blood is exposed to foreign surfaces (perhaps including xenotransplantation). Here, shortterm inhibition of complement may prove efficacious. However, if longterm inhibition of complement can be achieved safely and economically, the inhibitors might find a much wider clinical role. This will undoubtedly require the design of cheaper inhibitors with improved activity and duration of action in vivo. This rapidly moving area should convince even the most jaded of immunologists that complement research is no backwater but, rather, constitutes an exciting frontier of therapeutic immunology.

B. Paul Morgan is at the Dept of Medical Biochemistry, University of Wales College of Medicine, Heath Park, Cardiff, UK CF4 4XX.
References

1 Morgan, B.P. and Meri, S. (1994) Springer Semin. Immunopathol. 15, 369-416

2 Weisman, H.F., Bartow, T.,

Leppo, M.K. et al. (1990) Science 249, 146-150

3 Pepys, M.B. (1974) J. Exp. Med.

140, 126-145

4 Krych, M., Clemenza, L.,

Howdeshell, D. et al. (1994) J. Biol.

Chem. 269, 13273-13278

5 Vakeva, A., Jauhaianen, M.,

Ehnholm, C., Lehto, T. and Meri, S.

(1994) Immunology 82, 28-33

6 Chang, C-P., Husler, T., Zhao, J.,

Wiedmer, T. and Sims, P.J. (1994)

J. Biol. Chem. 269, 26424-26430

7 Platt, J.L., Vercellotti, G.M.,

Dalmasso, A.P. et al. (1990) Immunol.

Today 11, 450-456

8 White, D.J.G. (1992) Int. Arch.

Allergy Appl. Immunol. 98, 1-5

9 Morgan, B.P. (1994) Eur. J. Clin.

Invest. 24, 219-228

\title{
T-cell autoimmunity in multiple sclerosis
}

\section{Reinhard Hohlfeld, Marco Londei, Luca Massacesi and Marco Salvetti}

Multiple sclerosis (MS) is one of the most frequent causes of neurological impairment in young adults. Although the etiology of MS is still unknown, there is a general agreement that $\mathrm{MS}$ is an immunemediated disease of the central nervous system in which myelin is destroyed. Recently, innovative immunotherapies, ranging from inhibitory cytokines or anti-cytokines to vaccination with autoreactive $\mathrm{T}$ cells or T-cell receptors (TCRs), have raised hopes for the first time that this disorder can be treated. These novel therapies are based on the principle that an autoimmune T-cell response is a central element in the immunopathogenesis of MS.

\section{Search for new autoantigens}

One of the central issues in basic MS research is the identification of the T-cell autoantigen(s). For many years, myelin basic protein (MBP) was considered to be the only major autoantigen for experimental autoimmune encephalomyelitis (EAE)

\begin{abstract}
Autoreactive $T$ cells are crucial in the immunopathogenesis of multiple sclerosis. As discussed at a recent meeting *, research in this area has provided the basis for innovative immunotherapies.
\end{abstract}

and, accordingly, the only realistic candidate autoantigen for MS. Detailed studies of the human T-cell response against MBP have revealed that multiple MBP epitopes are recognized in the context of multiple restriction elements. Interestingly, in a minority of patients, $>80 \%$ of the T-cell clones selected with whole MBP respond to a patient-specific cluster of immunodominant epitopes. Such epitope clusters were noted in regions of the protein corresponding to residues $16-38$, 80-105, 108-131 and 131-153. In some patients, this reactivity pattern has remained remarkably stable over time (E. Meinl, Munich/Erlangen; M. Salvetti, Rome).

*The meeting 'T-Cell Autoimmunity in Multiple Sclerosis' was held in Rome, Italy, 21-22 January 1995.
In twins discordant for MS, the proliferative response to MBP appears to be higher in the affected twin (M. Londei, London). Furthermore, using single-cell cytokine-production assays (T. Olsson, Stockholm), peripheral blood mononuclear cells (PBMCs) and cerebrospinal fluid (CSF), cells from MS patients show increased responses to MBP and other myelin antigens, including proteolipid protein (PLP) and minor components such as myelin oligodendrocyte glycoprotein (MOG), which are encephalitogenic in animals ${ }^{1}$. In MS, the relative pathogenetic role of MBP, PLP, MOG and other myelin antigens remains to established.

One of the most interesting recent additions to the list of encephalitogenic autoantigens is $\mathrm{S} 100 \beta$, a calcium-binding protein of astroglia ${ }^{2}$. This is the first demonstration that a T-cell response against a nonmyelin autoantigen can induce EAE. Not surprisingly, there is now an intense search for T-cell responses 
against other myelin and nonmyelin autoantigens in MS $(\mathrm{H}$. van Noort, Leiden). However, the situation is complicated by the possibility that an initial T-cell response against a myelin or nonmyelin autoantigen triggers a subsequent $\mathrm{T}$ - (and B-) cell response against other CNS autoantigens (termed epitope spreading).

\section{Clues from TCR repertoire analyses}

As yet, it is not possible to demonstrate that human $T$ cells specific for CNS autoantigens in vitro are pathogenic in vivo. TCR repertoire studies may provide an indirect means to identify potentially pathogenic responses. PCR amplification and sequence analysis of mRNA isolated from MS brain revealed TCR sequences similar to the TCRs of MBP-specific T-cell clones isolated from the blood of MS patients $^{3}$. Furthermore, interleukin 2 receptor (IL-2R)-positive $\mathrm{T}$ cells freshly isolated from the peripheral blood of an MS patient, but never exposed to MBP in vitro, expressed the same TCR sequence as T-cell clones isolated from the same patient by stimulation with MBP (E. Meinl). These observations suggest that, at least in some MS patients, MBP is one of the autoantigens recognized in vivo. Clearly, this does not imply that MBP is the primary, or the only, autoantigen.

MS lesions contain $\gamma \delta$ as well as $\alpha \beta \mathrm{T}$ cells ${ }^{4}$. The $\gamma \delta \mathrm{T}$ cells in chronic, active MS lesions express a predominant V $\delta 2 J \delta 3$ gene rearrangement ${ }^{4}$. Experiments with T-cell lines derived from peripheral blood suggest that heat shock protein 70 (Hsp70) may select for $\mathrm{T}$ cells bearing the V $\delta 2 \mathrm{~J} \delta 3 \mathrm{TCR}$ (M. Salvetti). By contrast, mitogenstimulated $\gamma \delta \mathrm{T}$ cells, cultured from the CSF of MS patients, predominantly express Vס1 (G. DeLibero, Basle). Some of these CSF-derived $\gamma \delta$ T-cell clones kill glioblastoma and astrocytoma cell lines.

\section{New animal models}

It would be of great advantage if human autoimmune reactions could be studied in animal models. This may eventually be achieved using mice with multiple transgenes, expressing appropriate combinations of human cytokines, major histo- compatibility complex (MHC) and adhesion molecules, or with immunodeficient mice reconstituted with human immune cells. Alternatively, it may be possible to establish primate models in which human $\mathrm{T}$ cells interact with host cells in vivo. As a first step in this direction, it was demonstrated that a $\mathrm{CD}^{+}$HLA-DRB1 $* 0301$-restricted human T-cell clone can recognize MBP in vitro on PBMCs from different rhesus monkeys expressing closely related rhesus MHC alleles (Mamu-DRB1*0305 and DRB1* 0306) (B. t'Hart, Rijswijk; E. Meinl). In order to explore such 'trans-species models' further, and to characterize encephalitogenic $\mathrm{T}$ cells in primates, it will be helpful to establish novel EAE models in a variety of nonhuman primates, such as marmosets (L. Massacesi, Florence; B. t'Hart; A. Uccelli, Genoa).

\section{Role of cytokines: disease markers and targets for therapy?}

In the USA, the Food and Drug Administration (FDA) has recently approved a recombinant cytokine, interferon $\beta$ (IFN- $\beta$ ) $1 \mathrm{~b}$, as the first immunomodulatory treatment for MS (Ref. 5). This decision has greatly stimulated interest in the role of cytokines in the pathogenesis, diagnosis and therapy of MS. The exact mechanisms as to how IFN- $\beta$ mitigates MS are poorly understood, but are probably related to antagonistic effects on proinflammatory cytokines, such as IFN- $\gamma$ and tumour necrosis factor $\alpha$ (TNF- $\alpha$ ). Both of these cytokines are potent inducers of $\mathrm{MHC}$ class II antigens and adhesion molecules in target tissues. In MS patients (but not in healthy subjects), IFN- $\gamma$ stimulates $\mathrm{Ca}^{2+}$ influx in $\mathrm{CD}^{+}$ $T$ cells (G. Martino, Milan). This effect may be due to expression of a novel membrane channel, indicating that the $\mathrm{T}$ cells in MS are pre-activated.

Two proinflammatory cytokines thought to play a particularly prominant role in the development of MS lesions are TNF- $\alpha$ and TNF- $\beta$ (lymphotoxin) (K. Selmaj, Lodz). Treatment with soluble p55 TNF receptor is effective in EAE, and will probably soon be tested in MS. Other treatment strategies rely on cytokines that induce a shift from the pathogenic T helper 1 (Th1)-like response to a dominant Th2-like immune reaction ( $T$. Olsson).

Not only are cytokines (and soluble cell adhesion molecules) potentially useful as therapeutic agents, but also as markers of disease activity (P. Rieckmann, Göttingen; S. Jung, Würzburg; P. Gallo, Padua). Using a semiquantitative PCR technique, it was demonstrated that there is a significant increase in the expression of TNF- $\alpha$ and TNF- $\beta$ in PBMCs prior to clinical relapse (P. Rieckmann). At the same time, there is often a decrease of potentially downregulatory cytokines such as transforming growth factor $\beta$ (TGF- $\beta$ ) and IL-10.

\section{Cell banks as tools for MS research}

It is hoped that, in the near future, most MS patients will be treated early in the course of their disease. Therefore, it will be increasingly difficult to collect fresh PBMCs from untreated patients. A repository of large amounts of frozen PBMCs collected through apheresis from untreated MS patients may help to circumvent this problem (L. Massacesi).

\section{Conclusion}

Although many important aspects of the immunopathogenesis of MS have been elucidated, much remains to be learned. Future progress will profit from continued national and multinational cooperation in the quest for improved therapies.

The meeting was supported by the European Concerted Action on T-Cell Autoimmunity in MS. The authors thank $H$. Wekerle and C. Linington for helpful comments.

Reinhard Hohlfeld is at the Dept of Neurology, University of Munich, D-81366 Munich, Germany, and the Dept of Neuroimmunology, MaxPlanck Institute, D-82152 Martinsried, Germany; Marco Londei is at the Kennedy Institute of Rheumatology, Charing Cross Sunley Research Centre, Hammersmith, London, UK W6 8LW; Luca Massacesi is at the Dept of Neurological and Psychiatric Sciences, University of Florence, I-50134 Florence, Italy; Marco Salvetti 
is at the Dept of Neurological

Sciences, Università 'La Sapienza', I-00185 Rome, Italy.

References

1 Linington, C., Berger, T., Perry, L. et al. (1993) Eur. J. Immunol. 23 ,
1364-1372

2 Kojima, K., Berger, T., Lassmann, H. et al. (1994) J. Exp. Med. 180, 817-829

3 Oksenberg, J.R., Panzara, M.A., Begovich, A.B. et al. (1993) Nature $362,68-70$
4 Battistini, L., Selmaj, K., Kowal, C. et al. (1995) Ann. Neurol. 37,

198-203

5 Report of the Quality Standards

Subcommittee of the American

Academy of Neurology (1994)

Neurology 44, 1537-1540

\title{
Targeting cancer with radiolabeled antibodies
}

\author{
David M. Goldenberg, Steven M. Larson, Ralph A. Reisfeld \\ and Jeffrey Schlom
}

A recent conference focused on the advances, challenges and prospects of radioimmunodetection (RAID)

For many years, the strategies of cancer imaging and therapy with radioactive antibodies have made slow but steady strides in becoming new methods and agents in cancer management. Imaging with radiolabeled antibodies, termed radioimmunodetection (RAID), relies on a positive ratio between $\mathrm{cpm}$ at the target to cpm in adjacent, normal tissues or circulating blood. In therapy with radioactive antibodies, termed radioimmunotherapy (RAIT), progress has been more modest because of the necessity to achieve high selective radiation doses to tumor sites yet maintain acceptable toxicity levels in normal organs, especially myelotoxicity. This article reports on the progress and prospects of using RAID and RAIT in the treatment of cancer.

\section{Radiochemistry}

C.F. Meares (Davis, CA) summarized recent progress in radiochemical techniques, including new methods for effective pre-labeling prior to chelation of monoclonal antibodies (mAbs), in order to avoid the mixtures of macrocyclic compounds that may occur during postconjugation labeling. O.A. Gansow (Bethesda, MD) discussed novel, dendrimer-based, polymetal chelates, and their protein conjugates, with emphasis on enhancing the metal-ion-carrying capacity of mAbs for more-effective RAIT and for improved magnetic resonance imaging (MRI) by use of a polyamidoamine dendrimer as the intermediate and radioimmunotherapy (RAIT) of cancer. Emphasis was given to the underlying sciences of radiochemistry, as well as its clinical and experimental applications.

carrier. Enhanced site-specific conjugation of bifunctional chelating agents [diethylenetriamine petaacetic acids (DTPAs)] to a natural glycosylation site on the light chain of $F\left(a b^{\prime}\right)_{2}$ fragment of the antilymphoma mAb LL2 has been achieved (S.G. Govindan, Morris Plains, NJ). The same effect was demonstrated with an engineered, glycosylated version of the light chain of the anti-carcinoembryonic antigen (CEA) mAb IMMU-14 [which had been humanized by grafting of the complementaritydetermining regions (CDRs)]. Labeling of this carbohydrate site with such diverse radionuclides as ${ }^{90} \mathrm{Y}$, ${ }^{99 \mathrm{~m}} \mathrm{Tc},{ }^{111} \mathrm{In},{ }^{186} \mathrm{Re}$ and ${ }^{188} \mathrm{Re}$ was achieved. L.F. Mausner (Upton, NY) introduced a new RAIT isotope, ${ }^{47} \mathrm{Sc}$, considered to be useful because of its favorable physical properties and its coordination chemistry for chelating to mAbs. The use of $\alpha$-particle emitters conjugated with recombinant, humanized, anti-CD33 mAb M195, for the potential treatment of myeloid leukemias, has produced

* The 5th Conference on Radioimmunodetection and Radioimmunotherapy of Cancer was held at Princeton, NJ, USA, on 6-8 October 1994. encouraging results in animals (D.A. Scheinberg, New York, NY). A. Holmberg (Uppsala) reported that dextranation of antibodies improved the labeling efficacy of ${ }^{99 \mathrm{~m}} \mathrm{Tc}$ to $84-94 \%$. ${ }^{99 \mathrm{~m}} \mathrm{Tc}$ labeling of amide-derivatized single-stranded DNA for eventual use as singlestranded RNA and DNA oligonucleotides was described by D.J. Hnatowich (Worcester, MA).

\section{Experimental targeting}

Experimental studies of antibody targeting were introduced by D.J. Buchsbaum (Birmingham, AL), with emphasis on increasing tumor localization and accretion of radiolabeled antibodies. These strategies included genetically engineered or biochemically modified antibodies, unlabeled antibody pre-dosing, antibody cocktails, regional administration, and upregulation of target antigens by modifiers of biological responses. Methods described to increase the flow of blood through the tumor, or to increase its vascular permeability, resulted in higher tumor accretion of radiolabeled antibodies, and included external irradiation, hyperthermia and vasoactive drugs. Bifunctional antibodies enhanced clearance of nontargeted antibody from normal tissues and blood, and resulted in higher tumor: nontumor ratios.

Presentations on pre-targeting methods suggested that diverse methods for reducing targeting to normal tissues can enhance tumor radioimmunotherapy. D.B. Axworthy 